\title{
Editorial
}

\section{Amino acids in nutrition, health, and disease}

\author{
Guoyao $\mathrm{Wu}^{1, *}$ \\ ${ }^{1}$ Department of Animal Science, Texas A\&M University, College Station, TX 77843, USA
}

\section{TABLE OF CONTENTS}

\author{
1. Introduction \\ 2. Proline metabolism and signaling in parasites, bacteria and cancers \\ 3. Homeostasis as well as signaling and regulatory roles of AAs \\ 4. Functions of AAs in fetal growth and development \\ 5. Roles of AAs in intestinal metabolism, growth and health \\ 6. Experimental methods, models, and data analysis \\ 7. Impacts \\ 8. Author contributions \\ 9. Ethics approval and consent to participate \\ 10. Acknowledgments \\ 11. Funding \\ 12. Conflict of interest \\ 13. References
}

\section{Introduction}

Amino acids (AAs) are organic substances containing both amino and acid groups. Over 700 AAs occur in nature, and 20 of them serve as precursors for the synthesis of proteins in humans and other animals [1]. Except for glycine, AAs have either l- or d-isomers based on the chemical configuration of glyceraldehyde. Many of proteinogenic (protein-creating) AAs are utilized by tissues via cell-specific and multiple metabolic pathways to generate low-molecular-weight substances with enormous physiological importance, including creatine, nitric oxide (NO), polyamines, glutathione, heme, homoarginine, thyroid hormones, dopamine, and serotonin [2-9]. In addition, intestinal microbes (particularly those in the hindgut) can convert dietary AAs into ammonia, short-chain fatty acids (SCFAs), $\mathrm{H}_{2} \mathrm{~S}$, indole, and tryptamine that influence gut homeostasis and health [10]. In the whole body, AAs and their metabolites play important roles in cell signaling, gene expression, food intake, nutrient digestion and metabolism, immune and anti-oxidative responses, acid-base balance, hormone secretion, cognitive function and behavior, blood flow, osmoregulation, ammonia detoxification, and reproduction [1]. Thus, AAs are vital to the growth, development, health, and survival of humans and other animals. Some of these advances in AA biochemistry, physiology, and nutrition were highlighted in a special issue of Frontiers in Bioscience-Landmark entitled "Amino Acids in Nu- trition, Health, and Disease” [11-45]. Its specific topics included: (a) proline metabolism and signaling in parasites, bacteria and cancers [11-16]; (b) homeostasis as well as signaling and regulatory roles of AAs [17-26]; (c) functions of AAs in fetal growth and development [27-29]; (d) roles of AAs in intestinal metabolism, growth and health [30-37]; and (e) experimental methods, models, and data analysis [38-45].

\section{Proline metabolism and signaling in parasites, bacteria and cancers}

Proline contributes to interactions between microbial pathogens and their hosts through regulating oxidative stress, osmotic stress, cell signaling, and cell metabolism [12-15]. Thus, much work has been done to determine proline transport and metabolism in microbes and bacteria. Bringaud et al. [11] reported that trypanosomatids [(unicellular eukaryotic parasitic protozoans), such as Trypanosoma brucei subspecies (causing the sleeping sickness in Africa), Trypanosoma cruzi (causing Chagas disease), and Leishmania spp. (causing leishmaniasis)] are transmitted by blood-feeding arthropods. These insect vectors of the parasites use proline as a principal carbon and energy source, and transmit the parasites to humans through biting. Parasites transport proline across the cell membrane along a proton electrochemical gradient via protein carriers that are shared with alanine, cysteine, and hydroxypro- 
line. $\mathrm{K}_{M}$ values of the membrane transport for extracellular proline are 18.7, 310, and $60 \mu \mathrm{M}$ in Trypanosoma brucei, Trypanosoma cruzi, and Leishmania major, respectively [11]. The insect forms of trypanosomatids, such as T. brucei and T. congolense procyclics, T. cruzi epimastigotes, Leishmania spp. Promastigotes, and Crithidia spp. choanomastigotes, utilize proline as a major metabolic fuel in an age- and substrate-dependent manner [14]. For example, when trypanosomes develop into a procyclic form in the tsetse fly midgut where glucose is scarce, their procyclics make efficient uses of proline as a main energy source. By contrast, when glucose becomes abundant, trypanosomes switch to preferentially utilize glucose for ATP production because this sugar inhibits proline oxidase activity [14]. In all these parasites, proline dehydrogenase (a mitochondrial FAD-dependent enzyme) oxidizes proline to $\Delta^{1}$-pyrroline-5-carboxylate (P5C), which is further converted into ornithine, glutamate, and $\mathrm{CO}_{2}$ and, to a much lesser extent, acetate and ß-hydroxybutyrate. Parasites also express proline racemase for interconverting free l-proline into free d-proline possibly for the synthesis of d-prolinecontaining proteins and polypeptides. Reducing the conversion of ornithine into polyamines through the oral administration of dl- $\alpha$-difluoromethylornithine (DFMO; a specific enzyme-activated irreversible inhibitor of ornithine decarboxylase) is an effective therapy for the African sleeping sickness disease.

Microbes take up proline through several transmembrane protein carriers. For example, E. coli express three l-proline transport systems (PutP, ProP, and ProU) that differ in substrate specificity, the mechanism of energy coupling, and physiological roles [15]. PutP [a member of the $\mathrm{Na}^{+}$/solute symporter family (TC 2A.21, SLC5) with a stoichiometry of 1:1] is a high affinity, l-proline-specific transporter and regulates l-proline catabolism. ProP (a less specific transporter) transports l-proline and betaines with a low affinity, and acts as both an osmosensor and a transporter. ProU transports l-proline and betaines, and plays a role in the adaption of cells to osmotic stress. Both PutP and ProP are secondary transporters, whereas ProU is an ATP-binding cassette (ABC)-type transporter. In some bacteria, such as E. coli and Salmonella typhimurium, proline dehydrogenase and P5C dehydrogenase are fused into a bifunctional enzyme known as proline utilization A (PutA) that sequentially converts proline into glutamate. Microbial proline dehydrogenase uses FAD as an electron acceptor. Using the crystal structure of the minimalist PutA from Bradyrhizobium japonicum (BjPutA) along with sequence analysis, Singh and Tanner [12] identified the unique structural features of PutAs. Some PutAs have a 17-residue conserved motif in the C-terminal 20-30 residues of the polypeptide chain, and in the case of BjPutA, such a motif helps seal the internal substrate-channeling cavity from the bulk medium. In addition, some PutAs have a 100-200 residue domain with unknown function in the C-terminus.
These secondary structural elements and domains in PutAs, which are absent from the related monofunctional enzymes (i.e., separate proline dehydrogenase and P5C dehydrogenase), may facilitate substrate channeling for proline catabolism in Bradyrhizobium japonicum [12], as well as Pseudomonas putida and E. coli [13].

In mammals, proline oxidase (a mitochondrial enzyme; also known as proline dehydrogenase 1) uses ferricytochrome $\mathrm{C}$ as an electron acceptor to oxidize proline to P5C [16]. Another product of this enzymatic reaction is superoxide anion $\left(\mathrm{O}_{2}{ }^{-}\right)$, which can be converted into $\mathrm{H}_{2} \mathrm{O}_{2}$ and other reactive oxygen species with both physiological and pathological significance. P5C may be oxidized to glutamate by P5C dehydrogenase (a mitochondrial enzyme) or recycled to proline by $\mathrm{P} 5 \mathrm{C}$ reductase (present in mitochondria and the cytosol). In contrast to some bacteria noted previously, proline dehydrogenase and $\mathrm{P} 5 \mathrm{C}$ dehydrogenase are two different proteins in eukaryotes. In healthy mammalian cells and tissues (e.g., enterocytes and placenta), water is produced from proline oxidation. There is evidence that proline oxidation contributes to ATP production, while regulating protein and nucleotide syntheses, anaplerosis, and redox homeostasis in cancer cells [16]. Interestingly, proline oxidase is among a few genes that are induced rapidly and robustly by p53 (the tumor suppressor). A microRNA (miR-23b*), which is expressed at high levels in tumors, is a potent inhibitor of proline oxidase expression. Thus, proline catabolism may be a target for cancer therapy [16].

\section{Homeostasis as well as signaling and regulatory roles of AAs}

AAs in plasma have multiple metabolic fates: the syntheses of proteins, peptides, and low-molecular-weight substances, as well as oxidation to $\mathrm{CO}_{2}$ through inter-organ metabolism and cell-specific pathways. At a given developmental stage, the concentrations of free AAs in organs, tissues, cells, and plasma are relatively constant in healthy individuals in the post-absorptive state, due to the wellcontrolled balance between the supply (exogenous plus endogenous) and utilization of AAs [17]. Most AAs (except for arginine in the mammalian liver) are present in cells and tissues at much greater concentrations than those in the plasma. Notably, the concentrations of free AAs in the plasma and tissues vary with species, developmental stage, nutritional state, endocrine status, physical activity, time of the day, and diseased condition. In animal cells, the mechanistic target of rapamycin (MTOR; a highly conserved serine/threonine protein kinase) is a master activator of protein synthesis and cytoskeleton remodeling, as well as a negative regulator of protein degradation via autophagy [18-21]. The MTOR system consists of MTOR complexes 1 and 2, which are structurally and functionally distinct. AAs signal to MTOR complex 1 through the lysosomeassociated Rag GTPases. For example, leucine [18, 21, 23], 
glutamine [19], and arginine [20, 22] stimulate the phosphorylation of MTOR complex 1 to promote protein synthesis in many types of cells and tissues, including enterocytes, mammary epithelial cells, brown adipocytes, and skeletal muscle. The activated MTOR (i.e., phosphorylated MTOR) phosphorylates ribosomal protein S6 kinase-1 (S6K1) and eukaryotic translation initiation factor 4E (eIF4E)-binding protein-1 (4E-BP1, a translational repressor protein). The phosphorylation of 4E-BP1 reduces its binding affinity for eIF4E, thereby relieving the translational repression of $4 \mathrm{E}$ BP1 on the binding of eIF4E to eukaryotic translation initiation factor 4G (eIF4G) and generating the translationallyactive eIF4F-eIF4G complex to initiate the formation of protein. In addition, the phosphorylation of S6K1 results in hyperphosphorylation of ribosomal protein S6, thereby facilitating the translation of mRNAs for protein synthesis. These biochemical mechanisms explain why dietary supplementation with appropriate amounts of leucine, glutamine, and arginine to postnatal animals increases the use of dietary AAs for the protein accretion and growth of tissues (e.g., the small intestine and skeletal muscle), while reducing the partitioning of these nutrients into the syntheses of fatty acids and triacylglycerols in white adipose tissue. This also explains improved milk production by lactating mammals (e.g., swine) supplemented with these functional AAs [18-24].

Proteins synthesized from AAs are not only structural components of cells and tissues but are also regulators of gene expression and immunity in animals [19, 25]. Some of these proteins are octamer-binding proteins, which are a group of highly conserved transcription factors. These proteins bind to the octamer motif (ATGCAAAT) and closely related sequences in promoters and enhancers of a wide variety of target genes. As members of the family of Pit-Oct-Unc (POU) domain (DNA binding domain) factors, octamer-binding proteins possess an amino-terminal specific subdomain and a carboxylterminal homeo-subdomain. To date, eleven octamerbinding proteins have been identified. Octamer-binding proteins 1 and 2 are widely expressed in adult tissues, but other octamer-binding proteins are restricted to certain cell types. There is evidence that octamer-binding proteins affect a plethora of physiological events, including responses to stress, the proliferation and differentiation of taste cells, glucose and AA metabolism, embryogenesis, neurogenesis, immunity, and lactation. Defects in the expression of octamer-binding proteins result in various diseases, such as deafness, diabetes and cancer.

Besides their effects on gene expression and protein phosphorylation, AAs influence the secretion of some hormones from endocrine glands [25]. For example, leucine stimulates the secretion of insulin from pancreatic $\beta$-cells through the allosteric activation of glutamate dehydrogenase, resulting in elevated mitochondrial oxidative metabolism, ATP production, and membrane depolariza- tion. In addition, arginine promotes the release of insulin from pancreatic $\beta$-cells through the transport of this AA into the cells and the subsequent increase in membrane depolarization, leading to the opening of voltage-gated $\mathrm{Ca}^{2+}$ channels in the cell membrane and a rise in intracellular $\mathrm{Ca}^{2+}$ concentration. Furthermore, food-derived glutamate activates the vagus nerve and intestinal movement via the secretion of NO and serotonin from the gut, whereas glutamine inhibits the release of glucocorticoids (e.g., cortisol in swine; catabolic hormones) from the adrenal gland. Conversely, low protein intake (e.g., in growing and gestating rats) impairs the secretion of insulin, but ghrelin (a hormone released by enteroendocrine cells of the gastrointestinal tract) does not have such an effect [26].

\section{Functions of AAs in fetal growth and development}

In mammals, there are rapid increases in both placental and fetal growth, as well as the expansion of allantoic and amniotic fluids during gestation [27-29]. The accumulation of water within the conceptus requires the expression of aquaporins (AQPs) in the uterus, placenta and endometrium. AQPs are a family of small integral membrane proteins that primarily transport water across the plasma membrane, and some of them may transport glycerol, urea, ammonia, and small anions [29]. To date, thirteen AQP isoforms (AQP 0-12) have been identified in mammals, with AQPs 1, 2, 3, 4, 5, 6, 7, 8, 9, and 11 being identified in the female reproductive tract and in many other tissues. Expression of AQPs in the uterus and placenta is upregulated by hormones (progesterone and estrogen) and nutrients (e.g., arginine) to maintain fluid homeostasis in the conceptus via multiple signal transduction pathways mediated by cAMP, MAPK, PKC, and PI3 kinase/Akt/MTOR.

Many mammals (including humans, rats, cattle, and sheep) contain brown adipose tissue [27]. The sheep has provided a valuable model for understanding the fetal development of brown adipose tissue. This tissue has a high metabolic rate and expresses uncoupling protein-1. Maternal supplementation with arginine to ewes enhanced the mass and function of brown adipose tissue in fetal lambs. The underlying mechanisms include the production of NO and polyamines, as well as the expression of peroxisome proliferator-activated receptor (PPAR) gamma coactivator$1 \alpha$ (a transcription factor promoting mitochondrial biogenesis) and the activation of the MTOR cell signaling. As a result, newborn lambs from arginine-supplemented ewes have a greater amount of brown adipose tissue at birth, as well as greater rates of thermogenesis and survival during the neonatal period.

Conceptus survival and growth as well as pregnancy outcomes critically depend on placental angiogenesis, size, and function [26]. Among litter-bearing livestock mammals, swine exhibit the highest rates of embry- 
onic mortality (up to 50\%) and intrauterine growth restriction (IUGR; up to $25 \%$ in a litter). In pigs with the gestation length of 114 days, IUGR is associated with a reduced number of total muscle fibers in IUGR fetal pigs on Days 90 and 110 of gestation, when compared with fetal pigs with a normal body weight [28]. In addition, $27 \%$ of normal embryos are lost during the peri-implantation period in pregnant women, and $10-15 \%$ of surviving fetuses suffer from growth restriction worldwide. Thus, developing nutritional means to preventing embryonic death and IUGR is of enormous importance in both animal agriculture and human medicine. Notably, dietary supplementation with arginine beginning on Day 14 of gestation provides an effective strategy to maximize the number of live-born piglets per mother, increase the ratio of secondary-to-primary muscle fibers in fetal pigs, and alleviate IUGR in human fetuses.

\section{Roles of AAs in intestinal metabolism, growth and health}

The small intestine is a highly differentiated and complex organ with many functions. First, it is responsible for the terminal digestion and absorption of dietary nutrients and is, therefore, essential to health, growth, development, reproduction, and sustaining life in all animals [35]. Second, the neonatal small intestine can absorb immunoglobulins from milk before the "gut closure", which is important for the immunity of newborns. Third, the gut separates the internal milieu of the organisms from their external environment; therefore, it is critical for the exclusion of foodborne pathogens and preventing the translocation of luminal microorganisms into blood [30]. Fourth, in humans, pigs, and possibly other mammals, the small intestine extensively and selectively degrades some dietary AAs at various rates, such that approximately 95\%, 95\%, 70\%, 40\%, and 35$40 \%$ of dietary glutamate, aspartate, glutamine, proline, and branched-chain AAs do not enter the portal circulation [30]. Finally, as the largest lymphoid organ in the body, the small intestine participates in the immune surveillance of the intestinal epithelial layer and the regulation of mucosal responses to foreign antigens. Compared to the small intestine, the large intestine harbors much larger amounts of microorganisms that actively ferment carbohydrates, proteins, and AAs to SCFAs, $\mathrm{H}_{2} \mathrm{~S}$, ammonia, indoles, skatole, and other metabolites [31]. Interestingly, the hindgut is more susceptible to the development of tumors than the foregut.

Many dietary factors positively improve the integrity and function of the small intestine under the conditions of stress, such as weaning and immunological challenges [30-37]. These factors include $\alpha$-ketoglutarate [32], oleum cinnamomi [34], N-acetyl-cysteine [35], trilactic glyceride [37], arginine [22], and glutamine [19]. The underlying mechanisms responsible for their beneficial effects in the gut require the augmented syntheses of glutathione and polyamines, increased ATP production, the ac- tivation of anti-oxidative cell signaling pathway (including MTOR), increases in intestinal NO-dependent blood flow and oxygen supply, as well as the elevated expression of anti-inflammatory and tight-junction proteins in enterocytes [30-37]. Similar mechanisms may also apply to the large intestine.

\section{Experimental methods, models, and data analysis}

The recent decade has witnessed the rapid development of high-throughput and high-efficient technologies (e.g., genomics, transcriptomics, metabolomics, proteomics, bioinformatics, systems biology, and epigenetics) in AA nutrition research [38-40]. A goal of advanced nutrition research is to: (a) provide insights into the mechanisms whereby AAs and other nutrients influence the expression and biological activities of DNAs, RNAs, proteins, and low-molecular weight metabolites; and (b) define optimal requirements of individuals (e.g., humans as well as farm and laboratory animals) for AAs and other nutrients. Specifically, the proteomics technology can simultaneously determine thousands of proteins in a study sample (e.g., skeletal muscle and liver) and can identify their changes in response to physiological, pathological, and nutritional alterations. The most commonly used proteomics methods are based on either the specific digestion of proteins by trypsin or the direct analysis of intact proteins, followed by the chromatographic separation of peptides or intact proteins and their subsequent identification by mass spectrometry. Other quantitative proteomic techniques include 2D difference gel electrophoresis, isotope-coded affinity tag, isobaric tags for relative and absolute quantification, proteolytic O-18-labeling, and protein microarray technology. The metabolomics method allows for the analysis of the metabolome (a complete set of small-molecule metabolites in a biological sample) based on the proton nuclear magnetic resonance technology, mass spectrometry, and liquid chromatography-mass spectrometry. Functional outcomes of AA-induced changes in gene and protein expression as well as metabolite concentrations can be directly assessed by using appropriate experimental methods. Such approaches include Ussing chambers for studying nutrient transport by tissues (e.g., the small intestine and placentae) [29, 41] and indirect calorimetry for assessing whole-body energy expenditure and macronutrient oxidation [42].

Humans are members of the animal kingdom and share similarities to farm and laboratory animals in nutrition and metabolism. Biomedical research often involves invasive collections of tissues (e.g., the small-intestinal mucosa, liver, placenta, and kidney) and surgical procedures (e.g., the cannulation of the jugular vein, uterine artery and vein, and placental umbilical artery and vein) that may result in potential harmful effects, Thus, it is neither ethical nor practical to conduct such studies with human infants 
and adults. Historically, sheep have been used to elucidate mechanisms responsible for placental and fetal growth and development in humans [27], whereas pigs are invaluable for studying intestinal and cardiovascular physiology and health in humans [32-37, 39, 40, 43, 44], their nutrient requirements during the neonatal and lactation periods [18, 21], and their nutrient metabolism in the small and large intestines [30]. Of particular note, Hou and colleagues established three useful swine models for $E$. coli-induced neonatal diarrhea [43], indomethacin-induced intestinal injury [44], and oral administration of N-acetylcysteine to prevent intestinal dysfunction [35]. As detailed in the published studies [18, 27-29, 35], ovine and porcine models have both advantages and disadvantages in biomedical research and can be used to address important biological questions regarding human physiology and diseases. For comparison, rodents are commonly employed to study AA nutrition and metabolism due to their qualitative similarities to humans in biology, low costs, and the convenience of individual housing [22, 23, 26, 31, 42].

Statistical analysis is needed in biomedical research to make scientific inferences from data that contain variability [42, 45]. When a study involves only two populations (e.g., lean and obese rats [42]), the unpaired t-test is used for data analysis. When an experiment involves only one factor with three or more levels (e.g., 0, 20 and $40 \mathrm{~g}$ of supplemental protein in humans), data should be analyzed by one-way ANOVA (analysis of variance). When an experiment design involves two factors, such as dietary protein intake (low or high levels) as one factor and exercise status (low or high intensity) as another factor, two-way ANOVA must be used for data analysis. When an experiment design involves two factors (e.g., low- and high-fat diets) and repeated measurements (e.g., oxygen consumption) over different days, a mixed effect model that fits both fixed and random effects can be used for data analysis [42]. Likewise, the principal component analysis is powerful to reduce the dimensionality of a data set that consists of a large number of interrelated variables and to retain as much as possible the variation present in the data set [42]. Cluster analysis is a statistical tool designed for classification and data reduction purposes. This method allows for large data (e.g., the body weight of rats) to be classified into subgroups (e.g., normal or obese rats) based on their similar characteristics without prior knowledge of their group membership [42]. By contrast, when there is some prior knowledge of the potential number of subgroups within the given data set, the k-nearest neighbor procedure can be employed to classify relatively large data into subgroups.

Repeated-measures data are often generated from nutrition research. For example, in a study to evaluate the degradation of citrulline in the rumen of adult steers, the concentrations of citrulline in plasma are repeatedly measured from each animal at six different time points [45]. The objective of such an experiment is to determine whether time is an important factor in the mean changes of plasma citrulline concentrations. Accordingly, Lee et al. [45] described three mixed model analyses that can capture the time effect and account for the correlations of measurements over time from the same animals. These methods allow for flexible variance-covariance structures on the statistical model, the use of baseline measurements as a covariate in the model, and the use of percent-changes from baseline values for data normalization. All the three statistical approaches yield a meaningful conclusion that rumenprotected citrulline can bypass the rumen to effectively enter the blood circulation [45].

\section{Impacts}

Papers published in the special issue of Frontiers in Bioscience-Landmark entitled "Amino Acids in Nutrition, Health, and Disease” [11-45] have laid a framework for subsequent studies in this expanding research field. Examples include AA nutrition and metabolism in swine [4648], cattle [49-51], sheep [52-54], poultry [55-57], fish [58-63], crustaceans [64], and humans [65-67], as well as intestinal microbial AA metabolism $[68,69]$ and the modulation of proline metabolism for cancer therapy [70-72]. In addition, a much-needed database of all proteinogenic AAs plus key nonproteinogenic AAs and nitrogenous nutrients in common foodstuffs for humans and farm animals have recently been established [73-75]. Of note, the aforementioned articles in Frontiers in Bioscience-Landmark have contributed to (a) the use of functional AAs to improve the health, growth, food efficiency, reproduction, and productivity of farm animals [46-67] and (b) the development of nutritional means (e.g., oral or intravenous administration of glutathione, $\mathrm{N}$-acetyl-cysteine, arginine, and glutamine) to effectively alleviate severe syndromes of COVID-19 (one of the deadliest pandemics in history) in affected humans [76].

\section{Author contributions}

GW wrote this article and had the responsibility for its final content.

\section{Ethics approval and consent to participate}

Not applicable.

\section{Acknowledgments}

GW thanks the authors of papers in a special issue of Frontiers in Bioscience-Landmark entitled "Amino Acids in Nutrition, Health, and Disease” for their important contributions to the scientific development of this field. 


\section{Funding}

Work in the author's laboratory was supported by Agriculture and Food Research Initiative Competitive Grants (2015-67015-23276 and 2021-67015-34534) from the USDA National Institute of Food and Agriculture, as well as Texas A\&M AgriLife Research Hatch project (H8200).

\section{Conflict of interest}

The author declares no conflict of interest. GW is serving as one of the Editorial Board members of this journal. We declare that GW had no involvement in the peer review of this article and has no access to information regarding its peer review. Full responsibility for the editorial process for this article was delegated to GP.

\section{References}

[1] Wu G. Amino Acids: Biochemistry and Nutrition. 2nd edn. CRC Press: Boca Raton, Florida. 2021.

[2] Marc Rhoads J, Wu G. Glutamine, arginine, and leucine signaling in the intestine. Amino Acids. 2009; 37: 111-122.

[3] Phang JM, Liu W, Zabirnyk O. Proline metabolism and microenvironmental stress. Annual Review of Nutrition. 2010; 30: 441463.

[4] Bröer S, Bröer A. Amino acid homeostasis and signalling in mammalian cells and organisms. The Biochemical Journal. 2017; 474: 1935-1963.

[5] Hobbach AJ, Closs EI. Human cationic amino acid transporters are not affected by direct nitros(yl)ation. Amino Acids. 2020; 52: 499-503.

[6] Agostinelli E. Biochemical and pathophysiological properties of polyamines. Amino Acids. 2020; 52: 111-117.

[7] Neu A, Hornig S, Sasani A, Isbrandt D, Gerloff C, Tsikas D, et al. Creatine, guanidinoacetate and homoarginine in statin-induced myopathy. Amino Acids. 2020; 52: 1067-1069.

[8] Tsikas D, Bollenbach A, Hanff E, Beckmann B, Redfors B. Synthesis of homoagmatine and GC-MS analysis of tissue homoagmatine and agmatine: evidence that homoagmatine but not agmatine is a metabolite of pharmacological L-homoarginine in the anesthetized rat. Amino Acids. 2020; 52: 235-245.

[9] Manjarín R, Boutry-Regard C, Suryawan A, Canovas A, Piccolo $\mathrm{BD}$, Maj $\mathrm{M}$, et al. Intermittent leucine pulses during continuous feeding alters novel components involved in skeletal muscle growth of neonatal pigs. Amino Acids. 2020; 52: 1319-1335.

[10] Beaumont M, Blachier F. Amino acids in intestinal physiology and health. Advances in Experimental Medicine and Biology. 2020; 1265: 1-20.

[11] Bringaud F, Barrett MP, Zilberstein D. Multiple roles of proline transport and metabolism in trypanosomatids. Frontiers in Bioscience (Landmark Edition). 2012; 17: 349-374.

[12] Singh RK, Tanner JJ. Unique structural features and sequence motifs of proline utilization a (PutA). Frontiers in Bioscience (Landmark Edition). 2012; 17: 556-568.

[13] Arentson BW, Sanyal N, Becker DF. Substrate channeling in proline metabolism. Frontiers in Bioscience (Landmark Edition). 2012; 17: 375-388.

[14] Servet C, Ghelis T, Richard L, Zilberstein A, Savoure A. Proline dehydrogenase: a key enzyme in controlling cellular homeostasis. Frontiers in Bioscience (Landmark Edition). 2012; 17: 607-620.

[15] Jung H, Hilger D, Raba M. The $\mathrm{Na}^{+} /$L-proline transporter PutP.
Frontiers in Bioscience (Landmark Edition). 2012; 17: 745-759.

[16] Phang JM, Liu W. Proline metabolism and cancer. Frontiers in Bioscience (Landmark Edition). 2012; 17: 1835-1845.

[17] Liao SF, Regmi N, Wu G. Homeostatic regulation of plasma amino acid concentrations. Frontiers in Bioscience (Landmark Edition). 2018; 23: 640-655.

[18] Suryawan A, Davis TA. Regulation of protein synthesis by amino acids in muscle of neonates. Frontiers in Bioscience (Landmark Edition). 2011; 16: 1445-1460.

[19] Xi P, Jiang Z, Zheng C, Lin Y, Wu G. Regulation of protein metabolism by glutamine: implications for nutrition and health. Frontiers in Bioscience (Landmark Edition). 2011; 16: 578-597.

[20] Tan B, Li X, Yin Y, Wu Z, Liu C, Tekwe CD, et al. Regulatory roles for L-arginine in reducing white adipose tissue. Frontiers in Bioscience (Landmark Edition). 2012; 17: 2237-2246.

[21] Lei J, Feng D, Zhang Y, Zhao F, Wu Z, San Gabriel A, et al. Nutritional and regulatory role of branched-chain amino acids in lactation. Frontiers in Bioscience (Landmark Edition). 2012; 17: 2725-2739.

[22] Tan B, Xiao H, Xiong X, Wang J, Li G, Yin Y, et al. L-arginine improves DNA synthesis in LPS-challenged enterocytes. Frontiers in Bioscience (Landmark Edition). 2015; 20: 989-1003.

[23] Duan Y, Li F, Liu H, Li Y, Liu Y, Kong X, et al. Nutritional and regulatory roles of leucine in muscle growth and fat reduction. Frontiers in Bioscience (Landmark Edition). 2015; 20: 796-813.

[24] Zhao F. Octamer-binding transcription factors: genomics and functions. Frontiers in Bioscience (Landmark Edition). 2013; 18: 1051-1071.

[25] Newsholme P, Abdulkader F, Rebelato E, Romanatto T, Pinheiro CHJ, Vitzel KF, et al. Amino acids and diabetes: implications for endocrine, metabolic and immune function. Frontiers in Bioscience (Landmark Edition). 2011; 16: 315-339.

[26] Gao H, Ho E, Yallampalli C. Ghrelin doesn't limit insulin release in pregnant rats fed low protein diet. Frontiers in Bioscience (Landmark Edition). 2017; 22: 1523-1533.

[27] Satterfield MC, Wu G. Brown adipose tissue growth and development: significance and nutritional regulation. Frontiers in Bioscience (Landmark Edition). 2011; 16: 1589-1608.

[28] Wang T, Liu C, Feng C, Wang X, Lin G, Zhu Y, et al. IUGR alters muscle fiber development and proteome in fetal pigs. Frontiers in Bioscience (Landmark Edition). 2013; 18: 598-607.

[29] Zhu C, Jiang Z, Bazer FW, Johnson GA, Burghardt RC, Wu G. Aquaporins in the female reproductive system of mammals. Frontiers in Bioscience (Landmark Edition). 2015; 20: 838-871.

[30] Dai Z, Wu G, Zhu W. Amino acid metabolism in intestinal bacteria: links between gut ecology and host health. Frontiers in Bioscience (Landmark Edition). 2011; 16: 1768-1786.

[31] Blachier F, Davila AM, Benamouzig R, Tome D. Channelling of arginine in no and polyamine pathways in colonocytes and consequences. Frontiers in Bioscience (Landmark Edition). 2011; 16: 1331-1343.

[32] Hou Y, Wang L, Ding B, Liu Y, Zhu H, Liu J, et al. AlphaKetoglutarate and intestinal function. Frontiers in Bioscience (Landmark Edition). 2011; 16: 1186-1196.

[33] Wu X, Zhang Y, Yin Y, Ruan Z, Yu H, Wu Z, et al. Roles of heat-shock protein 70 in protecting against intestinal mucosal damage. Frontiers in Bioscience (Landmark Edition). 2013; 18: 356-365.

[34] Wang L, Hou Y, Yi D, Ding B, Zhao D, Wang Z, et al. Beneficial roles of dietary oleum cinnamomi in alleviating intestinal injury. Frontiers in Bioscience (Landmark Edition). 2015; 20: 814-828.

[35] Hou YQ, Wang L, Yi D, Wu G. N-Acetylcysteine and intestinal health: a focus on mechanisms of its actions. Frontiers in Bioscience (Landmark Edition). 2015; 20: 872-891.

[36] Yi D, Hou Y, Wang L, Zhao D, Ding B, Wu T, et al. Gene expression profiles in the intestine of lipopolysaccharide-challenged piglets. Frontiers in Bioscience (Landmark Edition). 2016; 21: 487-501.

[37] Hou Y. Trilactic glyceride regulates lipid metabolism and im- 
proves gut function in piglets. Frontiers in Bioscience (Landmark Edition). 2020; 25: 1324-1336.

[38] He Q, Yin Y, Zhao F, Kong X, Wu G, Ren P. Metabonomics and its role in amino acid nutrition research. Frontiers in Bioscience (Landmark Edition). 2011; 16: 2451-2460.

[39] Wu F, Xiong X, Yang H, Yao K, Duan Y, Wang X, et al. Expression of proteins in intestinal middle villus epithelial cells of weaning piglets. Frontiers in Bioscience (Landmark Edition). 2017; 22: 539-557.

[40] Xiong X, Yang H, Hu X, Wang X, Li B, Long L, et al. Differential proteome analysis along jejunal crypt-villus axis in piglets. Frontiers in Bioscience (Landmark Edition). 2016; 21: 343-363.

[41] He L, Yin Y, Li T, Huang R, Xie M, Wu Z, et al. Use of the Ussing chamber technique to study nutrient transport by epithelial tissues. Frontiers in Bioscience (Landmark Edition). 2013; 18: 1266-1274.

[42] Assaad H, Yao K, Tekwe CD, Feng S, Bazer FW, Zhou L, et al. Analysis of energy expenditure in diet-induced obese rats. Frontiers in Bioscience (Landmark Edition). 2014; 19: 967-985.

[43] Wu T, Lv Y, Li X, Zhao D, Yi D, Wang L, et al. Establishment of a recombinant Escherichia coli-induced piglet diarrhea model. Frontiers in Bioscience (Landmark Edition). 2018; 23: 15171534

[44] Yi D, Liu W, Hou Y, Wang L, Zhao D, Wu T, et al. Establishment of a porcine model of indomethacin-induced intestinal injury. Frontiers in Bioscience (Landmark Edition). 2018; 23: 21662176.

[45] Lee U, Garcia TP, Carroll RJ, Gillbreath KP, Wu G. Analysis of repeated measures data in nutrition research. Frontiers in Bioscience (Landmark Edition). 2019; 24: 1377-1389.

[46] Le Floc'h N, Wessels A, Corrent E, Wu G, Bosi P. The relevance of functional amino acids to support the health of growing pigs. Animal Feed Science and Technology. 2018; 245: 104-116.

[47] Zhang Q, Hou Y, Bazer FW, He W, Posey EA, Wu G. Amino acids in swine nutrition and production. Advances in Experimental Medicine and Biology. 2021; 1285: 81-107.

[48] Zhu C, Li X, Bazer FW, Johnson GA, Burghardt RC, Jiang Z, et al. Dietary L-arginine supplementation during days $14-25$ of gestation enhances aquaporin expression in the placentae and endometria of gestating gilts. Amino Acids. 2021; 53: 1287-1295.

[49] Bergen WG. Amino acids in beef cattle nutrition and production. Advances in Experimental Medicine and Biology. 2021; 1285: $29-42$.

[50] Gilbreath KR, Bazer FW, Satterfield MC, Wu G. Amino acid nutrition and reproductive performance in ruminants. Advances in Experimental Medicine and Biology. 2021; 1285: 43-61.

[51] Cao Y, Yao J, Sun X, Liu S, Martin GB. Amino acids in the nutrition and production of sheep and goats. Advances in Experimental Medicine and Biology. 2021; 1285: 63-79.

[52] Satterfield MC, Edwards AK, Bazer FW, Dunlap KA, Steinhauser CB, Wu G. Placental adaptation to maternal malnutrition. Reproduction. 2021; 162: R73-R83.

[53] McKnight SM, Simmons RM, Wu G, Satterfield MC. Maternal arginine supplementation enhances thermogenesis in the newborn lamb. Journal of Animal Science. 2020; 98: skaa118.

[54] Sales F, Sciascia Q, van der Linden DS, Wards NJ, Oliver MH, McCoard SA. Intravenous maternal arginine administration to twin-bearing ewes, during late pregnancy, is associated with increased fetal muscle mTOR abundance and postnatal growth in twin female lambs. Journal of Animal Science. 2016; 94: 2519 2531.

[55] He W, Li P, Wu G. Amino acid nutrition and metabolism in chickens. Advances in Experimental Medicine and Biology. 2021; 52: 109-131.

[56] He W, Furukawa K, Toyomizu M, Nochi T, Bailey CA, Wu G. Interorgan metabolism, nutritional impacts, and safety of dietary L-glutamate and L-glutamine in poultry. Advances in Experimental Medicine and Biology. 2021; 1332: 107-128.
[57] Furukawa K, He W, Bailey CA, Bazer FW, Toyomizu M, Wu G. Polyamine synthesis from arginine and proline in tissues of developing chickens. Amino Acids. 2021; 53: 1739-1748.

[58] Li X, Zheng S, Wu G. Nutrition and metabolism of glutamate and glutamine in fish. Amino Acids. 2020; 52: 671-691.

[59] Li X, Shixuan Zheng, Jia S, Song F, Zhou C, Wu G. Oxidation of energy substrates in tissues of largemouth bass (Micropterus salmoides). Amino Acids. 2020; 52: 1017-1032.

[60] Li XY, Zheng SX, Ma XK, Cheng KM, Wu G. Effects of dietary protein and lipid levels on growth performance, feed utilization, and liver histology of largemouth bass (Micropterus salmoides). Amino Acids. 2020; 52: 1043-1061.

[61] Li X, Zheng S, Ma X, Cheng K, Wu G. Effects of dietary starch and lipid levels on the protein retention and growth of largemouth bass (Micropterus salmoides). Amino Acids. 2020; 52: 999-1016.

[62] Li X, Zheng S, Wu G. Nutrition and functions of amino acids in fish. Advances in Experimental Medicine and Biology. 2021; 1285: 133-168.

[63] Palomino Ramos AR, Campelo DAV, Carneiro CLDS, Zuanon JAS, da Matta SLP, Furuya WM, et al. Optimal dietary Lglutamine level improves growth performance and intestinal histomorphometry of juvenile giant trahira (Hoplias lacerdae), a Neotropical carnivorous fish species. Aquaculture. 2022; 547: 737469 .

[64] Li X, Han T, Zheng S, Wu G. Nutrition and functions of amino acids in aquatic crustaceans. Advances in Experimental Medicine and Biology. 2021; 1285: 169-198.

[65] Wu G. Important roles of dietary taurine, creatine, carnosine, anserine and 4-hydroxyproline in human nutrition and health. Amino Acids. 2020; 52: 329-360.

[66] Ogilvie AR, Watford M, Wu G, Sukumar D, Kwon J, Shapses SA. Decreased glucogenic amino acids with a higher compared to normal protein diet during energy restriction in women: a randomized controlled trial. Amino Acids. 2021; 53: 1467-1472.

[67] Wu G, Meininger CJ, McNeal CJ, Bazer FW, Rhoads JM. Role of L-arginine in nitric oxide synthesis and health in humans. Advances in Experimental Medicine and Biology. 2021; 1332: 167-187.

[68] Dai Z, Wu Z, Zhu W, Wu G. Amino acids in microbial metabolism and function. Advances in Experimental Medicine and Biology. 2022; 1354: 127-143.

[69] Mu C, Pi Y, Zhang C, Zhu W. Microbiomes in the intestine of developing pigs: Implications for nutrition and health. Advances in Experimental Medicine and Biology. 2022; 1354: 161-176.

[70] Patel SM, Seravalli J, Stiers KM, Tanner JJ, Becker DF. Kinetics of human pyrroline-5-carboxylate reductase in L-thioproline metabolism. Amino Acids. 2021; 53: 1863-1874.

[71] Phang JM. Proline metabolism in cell regulation and cancer biology: Recent advances and hypotheses. Antioxidants \& Redox Signaling. 2019; 30: 635-649.

[72] Geng P, Qin W, Xu G. Proline metabolism in cancer. Amino Acids. 2021; 53: 1769-1777.

[73] Hou Y, He W, Hu S, Wu G. Composition of polyamines and amino acids in plant-source foods for human consumption. Amino Acids. 2019; 51: 1153-1165.

[74] Li P, Wu G. Composition of amino acids and related nitrogenous nutrients in feedstuffs for animal diets. Amino Acids. 2020; 52 523-542.

[75] Li P, Wu G. Functional molecules of intestinal mucosal products in animal nutrition and health. Advances in Experimental Medicine and Biology. 2022; 1354: 263-277.

[76] Li P, Wu G. Important roles of amino acids in immune responses. The British Journal of Nutrition. 2021. (in press)

Send correspondence to: Guoyao Wu, Department of Animal Science, Texas A\&M University, College Station, TX 77843, USA, E-mail: g-wu@tamu.edu 